\section{Overstating the lack of evidence on suicide risk assessment}

Chan and colleagues ${ }^{1}$ provide an overview of risk factors and risk scales for suicide following self-harm. However, their conclusions go beyond their review findings, and we think that discounting the potential value of risk scales on the basis of imperfect tools designed for other purposes is premature.

First, although we agree that the use of risk categories has its limitations (in particular when post hoc cut-offs are used), this can be resolved if risk prediction tools use pre-specified cut-offs, and consider reporting absolute probabilities as well as risk categories. ${ }^{2}$ Absolute probabilities provide greater flexibility, and could help optimise treatment allocation, waiting list prioritisation, or referral for more detailed assessments. A multicentre study in three UK centres found that only $70 \%$ of hospital episodes of self-harm receive psychosocial assessments, ${ }^{3}$ and hence there will likely be further challenges linking those at risk with appropriate clinical services. Clearly, psychosocial assessments are recommended for all persons who self-harm, but more personalised therapies will also involve a degree of triaging.

Second, the review identified three tools used in practice: the Beck Hopelessness Scale, Scale for Suicidal Ideation, and Suicide Intent Scale. However, none of these were developed for the purposes of risk prediction and thus critiquing the whole field on the basis of these tools goes beyond the evidence.

All risk prediction tools should be critically evaluated in terms of discrimination, calibration and reclassification - but the same high standards should also be applied to alternative approaches. What would be the performance of not using risk assessment, through purely qualitative or needs-based approaches? Without this information, this review might encourage a return to more subjective risk assessment approaches, which in the field of violence risk assessment have been shown to perform less well than structured methods. ${ }^{4}$

Although purely qualitative and needs-based approaches have a strong intuitive appeal, risk assessment, if it can be linked to treatment, is likely to play a part in reducing suicide risk.

\section{Declaration of interest}

A.W. is currently researching violence risk assessment. S.F. has published on violence risk assessment, including a tool (OxRec).

1 Chan MKY, Bhatti H, Meader N, Stockton S, Evans J, O'Connor RC, et al. Predicting suicide following self-harm: a systematic review of risk factors and risk scales. Br J Psychiatry 2016; 209: 277-83.

2 Fazel S, Chang Z, Fanshawe T, Långström N, Lichtenstein $\mathrm{P}$, Larsson $\mathrm{H}$, et al Prediction of violent reoffending on release from prison: derivation and external validation of a scalable tool. Lancet Psychiatry 2016; 3: 535-43.

3 Hawton K, Bergen H, Casey D, Simkin S, Palmer B, Cooper J, et al. Self-harm in England: a tale of three cities. Soc Psychiatry Psychiatr Epidemiol 2007; 42: 513-21.

4 AEgisdóttir S, White MJ, Spengler PM, Maugherman AS, Anderson LA, Cook RS, et al. The meta-analysis of clinical judgment project: fifty-six years of accumulated research on clinical versus statistical prediction. Couns Psychol 2006; 34: 341-82.

Achim Wolf, DPhil student and research assistant, Department of Psychiatry, University of Oxford. Email: achim.wolf@psych.ox.ac.uk; Seena Fazel, Wellcome Trust Senior Research Fellow, Department of Psychiatry, University of Oxford

doi: 10.1192/bjp.210.5.369

\section{The red corner: in support of formal risk prediction in psychiatry}

Assessing suicide risk is an integral part of what a mental health professional does. It permeates out even to the training programmes of charitable organisations such as Samaritans. So much emphasis is given to suicide risk assessment because suicide is one of the worst outcomes for lost years of life, and it has a ripple effect on surrounding family and friends that cannot be understated. However, predicting whether an individual will go on to die by suicide is a tricky business, and I sympathise with the rhetoric of Mulder and colleagues ${ }^{1}$ in a recent edition of the British Journal of Psychiatry. They rightly identify that because the base rate of suicide is so low in the general population (10.8 in 100000 ), our current formal predictive tools just do not achieve satisfactory statistical efficacy. I think it is appropriate to say not only that there is a base rate issue, but also that suicide risk is dynamic and that the validity of an initial assessment decays appreciably with increased follow-up time. All this stacks up against our attempts to formally risk assess patients. However, I would not support a retreat to relying purely on unstructured clinical judgement, for three reasons.

First, it is clear that humans, whether medically trained or not, are poor at predicting the future. This is not a new insight. 2016 marks 50 years since the Baxstrom $v$ Herold ruling. Essentially, this ruling meant that almost 1000 inmates who were previously regarded high risk for violent reoffending were released or had their confinement level stepped down. The controversy is that although they were believed to be high risk, very few went on to be reconvicted for violent crime. While acknowledging that suicide and violence are not analogous, this exemplifies the underlying flawed nature of unstructured professional judgement. Second, by incorporating structured professional judgement (SPJ) tools into our practice, we are not dissociating ourselves from the patient but following an evidence-based structure to inform our management. SPJs are conducted as interviews, leading to a numerical score which is flexible to interpretation by the clinician. Unlike when using atheoretical actuarial assessments, the clinician is not dictated to by the score. Unlike unstructured clinical judgement, the clinician can visualise all the relevant risk factors. Finally, to have a standardised approach is ideal for audit and reduces interclinician variability: a nod to the ideals of modern practice.

I was fortunate enough to be in the audience for a lecture by Professor Robert Snowden recently at a Royal College of Psychiatrists conference on old age psychiatry. He and his team are developing an SPJ tool, to be called the Risk of Suicide Protocol (RoSP), which may answer some of the issues we currently face in this area. Overall, given that suicide is such an important issue, with lifetime prevalence for attempt at nearly $3 \%$, can we afford to adopt a defeated rhetoric?

1 Mulder R, Newton-Howes G, Coid JW. The futility of risk prediction in psychiatry. Br J Psychiatry 2016; 209: 271-2.

\section{Edward Ackling, fourth-year medical student, Cardiff University. Email:} acklinge@cardiff.ac.uk

doi: 10.1192/bjp.210.5.369a

Authors' reply: We applaud Edward Ackling for his consideration of the importance of considering risks and agree that undertaking this difficult assessment is a core part of psychiatric practice. We also agree that suicide is 'one of the worst outcomes' and requires not only our clinical skills but our research interest. Ackling identifies the core issue of the problem of the low base rate, and again we agree, but we struggle to see how this provides a basis for suggesting that a structured risk assessment tool would be of benefit. The evidence to date simply does not support that stance. ${ }^{1}$ Ackling suggests the imprecision of future prediction supports a structured professional judgement (SPJ) tool and asserts that this 
allows us to remain associated to the patient while 'following an evidence base'. We would be interested to see the evidence base that such SPJ tools reduce rates of suicide in the general population, the population presenting to the emergency department for psychiatric review, or any day-to-day psychiatric population. To our knowledge, the only randomised controlled trial which compared SPJ with assessment as usual failed to show any superiority in preventing subsequent violence. ${ }^{2}$ Our concern is that SJP feels like science and is therefore more comfortable for the clinician, while not actually providing any clinical benefit.

The problem is that suicidal behaviour has multiple causes and occurs in many psychiatric conditions, as well as in the absence of any psychiatric diagnosis. There is nothing wrong with trying to create a structure for suicide risk assessment, but the emphasis on the predictive ability of SPJ (typically measured using the area under the curve (AUC) statistic) conceals the potentially endless list of risk factors that must be filtered through a clinical algorithm, and which ultimately only an experienced clinician can process. And for some diagnostic categories, reliance on SPJ to predict outcome is as accurate as tossing a coin. ${ }^{3}$

Ultimately, clinical experience and skill, rather than a checklist of commonly associated variables, are needed to determine the appropriate intervention. It is the intervention after assessment, or the risk management, that is the key and typically omitted from SPJ.

So, no more suicide risk assessments without risk management. It is also important to be wary of paying for courses taught by those with little relevant clinical experience such as the emergency room, as is often the case with SPJ. Finally, do psychiatrists who routinely assess suicide risk really carry out 'unstructured' assessments?

1 Chan MKY, Bhatti H, Meader N, Stockton S, Evans J, O'Connor RC, et al. Predicting suicide following self-harm: systematic review of risk factors and risk scales. Br J Psychiatry 2016; 209: 277-83.

2 Troquete NAC, van den Brink RHS, Beintema H, Mulder T, van Os TWDP, Schoevers RA, et al. Risk assessment and shared care planning in out-patient forensic psychiatry: cluster randomised controlled trial. Br J Psychiatry 2013; 202: $365-71$.

3 Coid J, Yang M, Tyrer P, Roberts A, Ullrich S. Prevalence and correlates of personality disorder in Great Britain. Br J Psychiatry 2006; 188: 423-31.

Roger T. Mulder, Professor of Psychological Medicine, University of Otago Christchurch, New Zealand. Email: roger.mulder@otago.ac.nz; Giles Newton-Howes Department of Psychological Medicine, University of Otago, Wellington, New Zealand; Jeremy Coid, Violence Prevention Unit, Wolfson Institute of Preventive Medicine, Queen Mary University, London, UK

doi: 10.1192/bjp.210.5.369b

\section{poems by doctors}

\section{Monochrome}

Francis Anthony O'Neill

We never had that frank discussion,

about the birds and bees,

or how the storm of sixty-five,

made that tree,

fall and cut your bus in two.

Your face in monochrome,

etched upon my brain

with Miss Hozier's husband on a barge.

We lay upon the bed

and watched the clouds float by,

the altitude determining their velocity,

and talked about how cartoons work,

and the power of metonymy. 